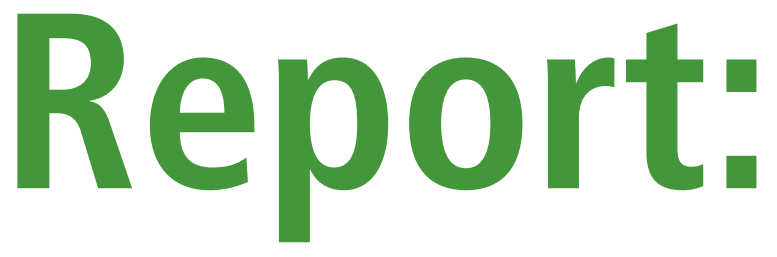

\title{
Live births in England and Wales, 2008: area of residence
}

This report provides provisional summary statistics of live births in England and Wales during 2008 and compares them with figures for previous years. It also presents provisional numbers and fertility rates by mother's usual area of residence. It is planned to publish further details of births in 2008 on the Office for National Statistics website (www.ons.gov.uk) throughout 2009 starting with parents' country of birth in August 2009.

\section{National}

- The Total Fertility Rate (TFR) in 2008 was 1.95 . This means women would have on average 1.95 children each if fertility rates at each age remained at 2008 levels in the future. The 2008 TFR represents the seventh consecutive annual increase from the low point in 2001 when the TFR was 1.63. The TFR is now at its highest level since $1973(2.00)$

- The General Fertility Rate (GFR) in 2008 was 63.5 live births per 1,000 women aged 15-44, an increase on the 2007 figure of 62.0 . This is a return to the level last seen in 1992 when it was 63.6

- There were 708,708 live births in England and Wales in 2008 compared with 690,013 in 2007 , an increase of 2.7 per cent

- There were increases in the fertility rates of women in all age groups in 2008 as compared to 2007 (see Figure 1). Women in their early 30 s continued to have the highest fertility rates, at 112.3 live births per 1,000 women aged 30 to 34 , having overtaken women in their late 20 s in 2004

- The average (mean) age of mothers at live birth has been rising steadily since the mid-1970s (see Figure 2). However, in 2008, the standardised mean age of mothers giving birth remained at 29.3 years, as in 2007. The standardised mean age of mothers at birth in 2008 was 2.9 years higher than the lowest mean age of 26.4 which was seen in 1974. See Explanatory Note 4

- Fertility rates for women aged 40 and over have been increasing over the past 20 years. In 2008, the provisional rate reached 12.6 live births per 1,000 women aged 40 and over, more than twice the 1988 rate of 5.1. Birth rates for women aged 40 and over are at their highest level since 1965 . There were 26,419 live births to mothers aged 40 and over in 2008, nearly double the 1998 figure $(13,555)$ and nearly treble the number in $1988(9,027)$
- In the decade since 1998 , the fertility rates of women in their late 30 s have increased by 45 per cent to 58.4 live births per 1,000 women aged 35-39 (see Figure 1)

- Over the past ten years, the fertility rates of women in their $20 \mathrm{~s}$ have fluctuated. However, in 2008 the fertility rates for women aged 20-24 were at their highest since 1998 at 74.3 live births per 1,000 women while rates for women aged 25-29 were at their highest since 1996 at 106.2 live births per 1,000 women

\begin{tabular}{|c|c|c|c|c|c|c|}
\hline Table 1 & Sum & nary of $k$ & ey live bir & th statist & ics, 1998- & 2008 \\
\hline \begin{tabular}{|l} 
Number of \\
live births
\end{tabular} & $\begin{array}{c}\text { Total } \\
\text { Fertility } \\
\text { Rate } \\
\text { (TFR) }\end{array}$ & $\begin{array}{c}\text { General } \\
\text { Fertility } \\
\text { Rate } \\
\text { (GFR): all } \\
\text { live births } \\
\text { per 1,000 } \\
\text { women } \\
\text { aged } \\
15-44\end{array}$ & \begin{tabular}{|c|} 
Sex ratio: \\
live male \\
births \\
per 1,000 \\
live female \\
births
\end{tabular} & $\begin{array}{c}\text { Mean } \\
\text { age of } \\
\text { mother at } \\
\text { childbirth } \\
\text { (years) }{ }^{2}\end{array}$ & $\begin{array}{c}\text { Percentage } \\
\text { of live } \\
\text { births } \\
\text { outside } \\
\text { marriage }\end{array}$ & $\begin{array}{c}\text { Percentage } \\
\text { of live } \\
\text { births } \\
\text { to non-UK } \\
\text { born } \\
\text { mothers }\end{array}$ \\
\hline 635,901 & 1.72 & 59.2 & 1,051 & 28.3 & 37.8 & 13.6 \\
\hline 621,872 & 1.70 & 57.8 & 1,055 & 28.4 & 38.9 & 14.3 \\
\hline 604,441 & 1.65 & 55.9 & 1,050 & 28.5 & 39.5 & 15.5 \\
\hline 594,634 & 1.63 & 54.7 & 1,050 & 28.6 & 40.0 & 16.5 \\
\hline 596,122 & 1.65 & 54.7 & 1,055 & 28.7 & 40.6 & 17.7 \\
\hline 621,469 & 1.73 & 56.8 & 1,051 & 28.8 & 41.4 & 18.6 \\
\hline 639,721 & 1.78 & 58.2 & 1,054 & 28.9 & 42.2 & 19.5 \\
\hline 645,835 & 1.79 & 58.3 & 1,049 & 29.1 & 42.8 & 20.8 \\
\hline 669,601 & 1.86 & 60.2 & 1,047 & 29.1 & 43.5 & 21.9 \\
\hline 690,013 & 1.92 & 62.0 & 1,057 & 29.3 & 44.3 & 23.2 \\
\hline 708,708 & $1.95^{3}$ & $63.5^{3}$ & 1,050 & 29.3 & 45.3 & 24.1 \\
\hline
\end{tabular}

1 The Total Fertility Rate is the average number of live children that a group of women would bear if they experienced the age-specific fertility rates of the calendar year in question throughout their childbearing lifespan.

2 The mean age of mother at childbirth is standardised. This measure eliminates the impact of any changes in the distribution of the population by age and therefore enables trends over time to be analysed.

3 Fertility rates for 2008 have been calculated using the 2006-based population projections for 2008.

p Figures for 2008 are provisional.

Source: Office for National Statistics (ONS), FM1 Tables 1.1a, 1.1b, 1.4, 1.7b and 9.1 


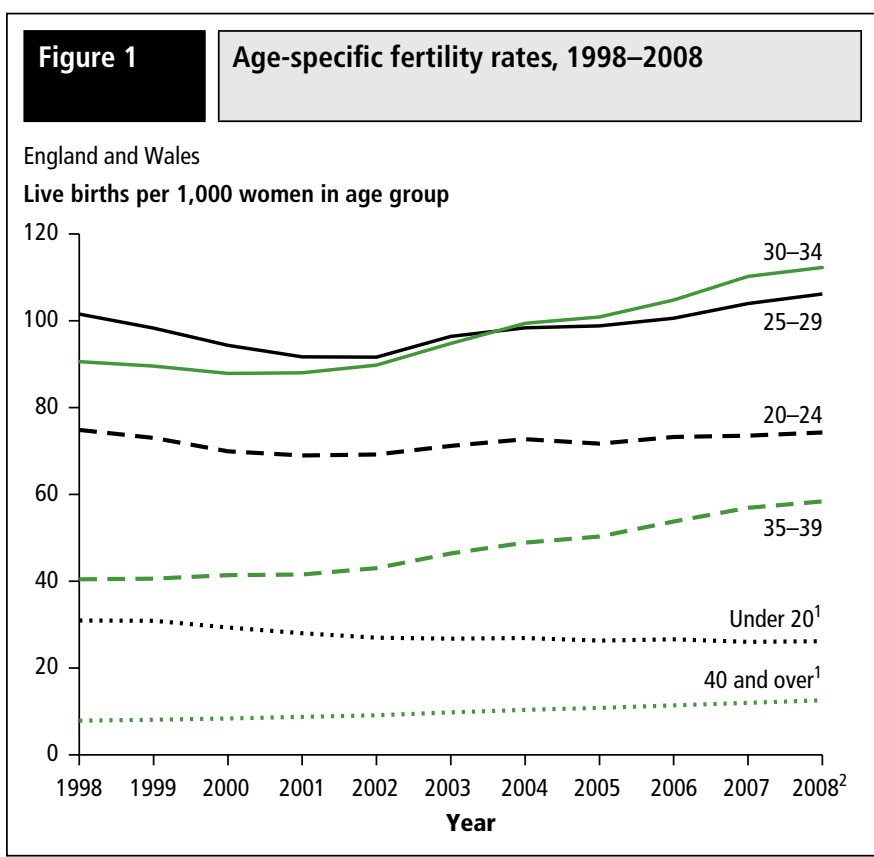

1 The rates for women aged under 20, and 40 and over, are based upon the population of women aged 15-19 and 40-44 respectively.

2 Figures for 2008 are provisional. Rates for 2008 are based on the 2006-based population projections for 2008 .

- The rise in the proportion of live births outside marriage continued: 44 per cent of live births were outside marriage in 2007, increasing to 45 per cent in 2008

- The proportion of live births to mothers born outside the UK continued to rise. In 2008, 24 per cent of live births in England and Wales were to mothers born outside the UK compared with 23 per cent in 2007 and 14 per cent in 1998

- In 2008, the number of live births to mothers born outside the UK was 170,833 compared with 537,852 live births to mothers born in the UK. Between 2007 and 2008 the proportionate increase in live births to mothers born outside the UK was greater than the proportionate increase in live births to mothers born in the UK. For mothers born outside the UK the number of live births rose by 6.5 per cent from 160,340 while for mothers born in the UK

\section{Figure 2 Standardised mean age of mother at live birth, 1968-2008}

England and Wales

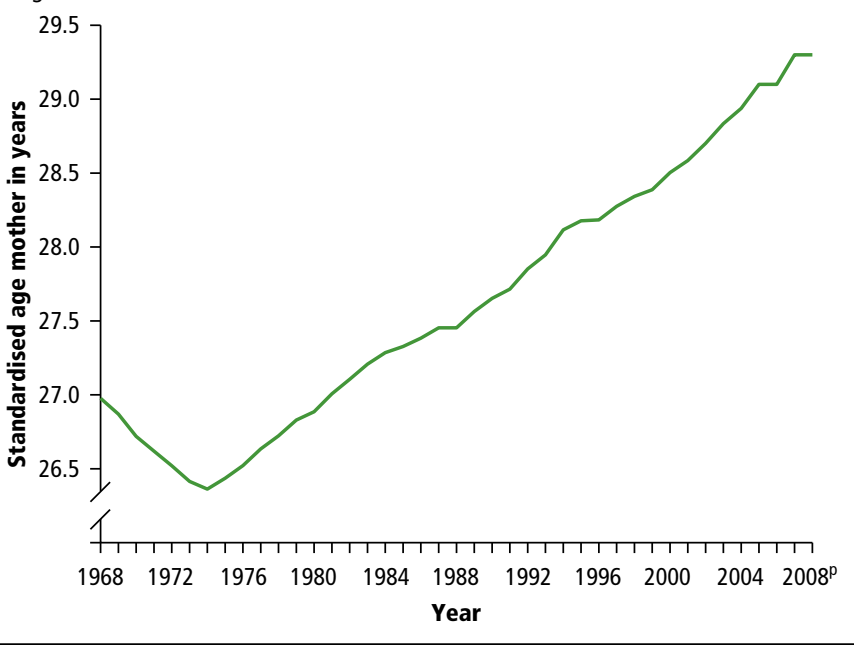

p Provisional

See Explanatory Notes 4 and 5. the number of live births rose by 1.5 per cent from 529,655 . See Explanatory Note 6

- The increase in the number of births in England and Wales to mothers born outside the UK is due partly to the rise in births to mothers born in countries belonging to the European Union. In 2007, there were 37,349 live births in England and Wales to mothers born in one of the other 26 member states of the European Union as constituted in 2007 (excluding the UK). This was 5.4 per cent of all live births that year. In 2008, this proportion increased to 6.1 per cent, with 43,444 live births to mothers born in the European Union (excluding the UK). See Explanatory Note 7 - The sex ratio at birth for 2008 was 1,050 live males per 1,000 live females born. This means that 5.0 per cent more boys than girls were born in 2008. This ratio has decreased from 1,057 live males per 1,000 live females in 2007 and normally fluctuates around this level

\section{Subnational}

Variations in fertility by area are shown in Tables $\mathbf{2}$ and $\mathbf{3}$, where provisional numbers of live births and provisional GFRs and TFRs are presented for administrative and health areas.

- The highest fertility level among the Government Office Regions of England in 2008, as represented by the TFR, was in the West Midlands where there was an average of 2.09 children per woman. The lowest was in the North East with 1.86. See Explanatory Note 8

- The highest level of fertility among the Government Office Regions of England in 2008, as represented by the GFR, was in London with 69.4 live births per 1,000 women aged 15-44, followed by the West Midlands (66.4). The lowest GFR was in the North East (58.7)

- The London borough of Newham recorded the highest GFR among local authorities in England with 96.5 live births per 1,000 women aged 15-44. The London borough of Barking and Dagenham and the unitary authority of Slough share the second highest GFR of 94.0. The lowest GFR was in Durham county district (39.4)

- Of the local authorities in England, Boston had the highest TFR of 2.81 children per woman, followed by Barking and Dagenham (2.80). The lowest TFR was in Westminster (1.21), followed by Camden (1.26)

- In Wales, the national GFR was 61.7 live births per 1,000 women aged 15-44 and the TFR was 1.96 children per woman. This is the first year that the TFR for Wales has been higher than that for England

- In Wales, the unitary authority with the highest GFR was Merthyr Tydfil (70.6); the highest TFRs were in Merthyr Tydfil and Newport which both recorded 2.30 children per woman. The lowest fertility was seen in Ceredigion where the GFR was 40.0 and the TFR was 1.43

\section{Explanatory Notes}

1. In this report, all figures (numbers and rates) for 2008 are provisional. At subnational level fertility rates have been calculated using mid-2007 population estimates and at national level the 2006-based population projections for 2008 have been used. The latter are available on the Government Actuary's Department website: www.gad.gov.uk/Population/index.asp. The population figures used to calculate fertility rates for 2007 and earlier years are the ONS mid-year population estimates. The population estimates used were the most up-to-date at the time of writing of this report. Further information on population estimates can be found on the Office for National Statistics website (www.statistics.gov.uk/popest).

2. Numbers of births, GFRs and TFRs are given by mother's usual area of residence, based on 2008 Local and Strategic Health Authority area boundaries (Local Health Boards in Wales). 
3. Provisional national TFRs have been calculated using the number of live births by single year of age. The provisional sub-national TFRs have been calculated using the number of live births by five year age groups.

4. The standardised mean age is a measure which eliminates the impact of any changes in the distribution of the population by age and therefore enables trends over time to be analysed.

5. Data collected on the number of births for 1981 were affected by a registrars' strike. Figures for this year are based on a 10 per cent sample of registrations.

6. Figures for live births to mothers born outside the UK and mothers born in the UK exclude births to mothers whose country of birth was not stated.

7. The percentage of births to mothers born in the European Union excludes births where the mother was born in the UK. There are 27 member states of the European Union. A full breakdown of the country groupings as constituted in 2008 can be found here: www.statistics.gov.uk/statbase/Product.asp?vlnk=14408 under FM1 Chapter 9: Parents' Birthplace.

8. The GFR and TFR show different results for fertility rate rankings by area. This is because the TFR controls for the age structure of the population while the GFR does not.

9. Further information on live births in 2008 can be found in Reference Tables 2.1 and 3.1 to 3.3 in this publication and at the births topic-based summary on the Office for National Statistics website: www.statistics.gov.uk/cci/nugget.asp?id=369

10. 2008 births data collected at registration contained 1.0 per cent of records where mother's date of birth was not stated. For records where mother's date of birth was not stated and the birth was successfully linked to birth notification records, the mother's date of birth was taken from this source ( 0.8 per cent of all births). Where linkage with the birth notification was unsuccessful, mother's age was imputed ( 0.2 per cent of all births). 
England and Wales, Government Office Regions (within England), unitary authorities/counties/districts \& London boroughs

\begin{tabular}{|c|c|c|c|c|c|c|c|}
\hline Area of usual residence & Live births & $\mathrm{GFR}^{1}$ & $\mathrm{TFR}^{2}$ & - continued & Live births & $\mathrm{GFR}^{1}$ & $\mathrm{TFR}^{2}$ \\
\hline \multirow[t]{2}{*}{ ENGLAND AND WALES } & 708,708 & 63.5 & 1.95 & Manchester & 7,749 & 65.9 & 1.91 \\
\hline & & & & Oldham & 3,289 & 74.2 & 2.35 \\
\hline ENGLAND & 672,807 & 63.6 & 1.95 & Rochdale & 3,043 & 73.0 & 2.32 \\
\hline \multirow[t]{2}{*}{ NORTH EAST } & 30,217 & 58.7 & 1.86 & Salford & 3,340 & 70.9 & 2.09 \\
\hline & & & & Stockport & 3,366 & 60.6 & 1.95 \\
\hline Darlington UA & 1,337 & 68.3 & 2.19 & Tameside & 2,936 & 65.5 & 2.12 \\
\hline Hartlepool UA & 1,164 & 63.4 & 2.05 & Trafford & 2,841 & 66.1 & 2.07 \\
\hline Middlesbrough UA & 1,891 & 65.2 & 2.01 & Wigan & 3,949 & 64.2 & 2.06 \\
\hline Redcar and Cleveland UA & 1,586 & 59.1 & 1.93 & & & & \\
\hline \multirow[t]{2}{*}{ Stockton-on-Tees UA } & 2,449 & 62.7 & 2.02 & Lancashire & 13,963 & 60.8 & 1.96 \\
\hline & & & & Burnley & 1,315 & 73.7 & 2.36 \\
\hline Durham & 5,686 & 57.0 & 1.83 & Chorley & 1,239 & 62.6 & 2.04 \\
\hline Chester-le-Street & 561 & 55.0 & 1.88 & Fylde & 642 & 51.0 & 1.80 \\
\hline Derwentside & 1,016 & 59.9 & 1.96 & Hyndburn & 1,159 & 70.9 & 2.28 \\
\hline Durham & 838 & 39.4 & 1.38 & Lancaster & 1,487 & 47.4 & 1.49 \\
\hline Easington & 1,177 & 62.7 & 2.01 & & & & \\
\hline \multirow{2}{*}{ Sedgefield } & 1,095 & 65.3 & 2.14 & Pendle & 1,322 & 74.6 & 2.37 \\
\hline & & & & Preston & 1,940 & 66.3 & 2.00 \\
\hline Teesdale & 218 & 55.7 & 1.98 & Ribble Valley & 505 & 48.1 & 1.72 \\
\hline \multirow[t]{2}{*}{ Wear Valley } & 781 & 65.7 & 2.21 & Rossendale & 876 & 65.1 & 2.16 \\
\hline & & & & South Ribble & 1,255 & 60.1 & 1.98 \\
\hline Northumberland & 3,094 & 56.4 & 1.90 & & & & \\
\hline Alnwick & 291 & 54.9 & 1.95 & West Lancashire & 1,243 & 59.5 & 1.97 \\
\hline Berwick-upon-Tweed & 169 & 42.3 & 1.47 & Wyre & 980 & 51.5 & 1.80 \\
\hline Blyth Valley & 916 & 57.6 & 1.83 & & & & \\
\hline Castle Morpeth & 442 & 55.6 & 1.95 & Merseyside (Met County) & 16,237 & 58.4 & 1.87 \\
\hline Tynedale & 537 & 54.7 & 1.95 & Knowsley & 1,981 & 61.0 & 2.01 \\
\hline \multirow[t]{2}{*}{ Wansbeck } & 739 & 62.3 & 2.04 & Liverpool & 5,595 & 56.4 & 1.73 \\
\hline & & & & Sefton & 2,718 & 52.7 & 1.82 \\
\hline Tyne and Wear (Met County) & 13,010 & 57.3 & 1.77 & St Helens & 2,156 & 60.9 & 1.99 \\
\hline Gateshead & 2,352 & 61.8 & 1.96 & Wirral & 3,787 & 64.0 & 2.12 \\
\hline Newcastle upon Tyne & 3,293 & 52.7 & 1.66 & & & & \\
\hline North Tyneside & 2,406 & 61.5 & 1.92 & YORKSHIRE AND THE HUMBER & 66,353 & 62.4 & 1.94 \\
\hline South Tyneside & 1,670 & 56.0 & 1.79 & & & & \\
\hline \multirow[t]{2}{*}{ Sunderland } & 3,289 & 57.1 & 1.79 & East Riding of Yorkshire UA & 3,064 & 52.9 & 1.84 \\
\hline & & & & Kingston upon Hull, City of UA & 3,682 & 65.0 & 1.88 \\
\hline \multirow[t]{2}{*}{ NORTH WEST } & 88,167 & 63.2 & 2.00 & North East Lincolnshire UA & 1,957 & 62.6 & 2.04 \\
\hline & & & & North Lincolnshire UA & 1,888 & 63.7 & 2.14 \\
\hline Blackburn with Darwen UA & 2,377 & 81.1 & 2.53 & York UA & 2,092 & 48.4 & 1.51 \\
\hline Blackpool UA & 1,745 & 64.9 & 2.12 & & & & \\
\hline Halton UA & 1,656 & 66.9 & 2.10 & North Yorkshire & 6,197 & 58.5 & 2.00 \\
\hline \multirow[t]{2}{*}{ Warrington UA } & 2,459 & 62.4 & 2.05 & Craven & 467 & 50.5 & 1.83 \\
\hline & & & & Hambleton & 871 & 59.3 & 2.09 \\
\hline Cheshire & 7,782 & 59.2 & 1.94 & Harrogate & 1,706 & 57.9 & 1.90 \\
\hline Chester & 1,322 & 54.2 & 1.75 & Richmondshire & 559 & 60.4 & 1.98 \\
\hline Congleton & 907 & 53.5 & 1.78 & Ryedale & 452 & 50.5 & 1.73 \\
\hline Crewe and Nantwich & 1,520 & 67.3 & 2.23 & & & & \\
\hline Ellesmere Port \& Neston & 959 & 60.9 & 1.99 & Scarborough & 1,126 & 60.7 & 2.07 \\
\hline Macclesfield & 1,638 & 59.3 & 1.90 & Selby & 1,016 & 64.5 & 2.25 \\
\hline \multirow[t]{2}{*}{ Vale Royal } & 1,436 & 59.7 & 2.01 & & & & \\
\hline & & & & South Yorkshire (Met County) & 16,498 & 61.9 & 1.93 \\
\hline Cumbria & 5,118 & 57.5 & 1.94 & Barnsley & 2,777 & 62.0 & 2.05 \\
\hline Allerdale & 983 & 58.9 & 2.01 & Doncaster & 3,804 & 67.7 & 2.20 \\
\hline Barrow-in-Furness & 815 & 58.7 & 1.93 & Rotherham & 3,263 & 65.3 & 2.11 \\
\hline Carlisle & 1,225 & 61.9 & 1.98 & Sheffield & 6,654 & 57.5 & 1.78 \\
\hline Copeland & 747 & 57.7 & 1.94 & & & & \\
\hline Eden & 472 & 54.2 & 1.91 & West Yorkshire (Met County) & 30,975 & 65.6 & 1.99 \\
\hline \multirow[t]{2}{*}{ South Lakeland } & 876 & 51.8 & 1.82 & Bradford & 8,580 & 81.5 & 2.42 \\
\hline & & & & Calderdale & 2,665 & 66.3 & 2.17 \\
\hline \multirow{2}{*}{$\begin{array}{l}\text { Greater Manchester } \\
\text { (Met County) }\end{array}$} & 36,830 & 67.5 & 2.06 & Kirklees & 5,814 & 70.0 & 2.18 \\
\hline & & & & Leeds & 9,844 & 55.0 & 1.68 \\
\hline Bolton & 3,873 & 72.7 & 2.33 & Wakefield & 4,072 & 62.7 & 2.05 \\
\hline Bury & 2,444 & 66.0 & 2.12 & & & & \\
\hline
\end{tabular}

Notes: Figures for 2008 are provisional. A birth to a mother whose usual residence is outside England and Wales is assigned to the country of residence. These births are included in total figures for England and Wales, but excluded from any sub-division of England and Wales.

1 The General Fertility Rate (GFR) is the number of live births per 1,000 women aged 15-44. The national GFRs have been calculated using 2006-based population projections for 2008 and the sub-national GFRs have been calculated using the 2007 mid-year population estimates.

2 The Total Fertility Rate (TFR) is the average number of live children that a group of women would bear if they experienced the age-specific fertility rates of the calendar year in question throughout their childbearing lifespan. The national TFRs have been calculated using the number of live births by single year of age and the 2006-based population projections for 2008. The sub-national TFRs have been calculated using the number of live births by five year age groups and the 2007 mid-year population estimates.

3 City of London has been grouped with Hackney, and Isles of Scilly grouped with Penwith, because of the very small number of births in these areas. 
Table 2

continued

Live births by local authority of usual residence of mother, numbers, General Fertility Rates and Total Fertility Rates, 2008

England and Wales, Government Office Regions (within England), unitary authorities/counties/districts \& London boroughs

\begin{tabular}{|c|c|c|c|c|c|c|c|}
\hline Area of usual residence & Live births & $\mathrm{GFR}^{1}$ & $\mathrm{TFR}^{2}$ & - continued & Live births & $\mathrm{GFR}^{1}$ & $\mathrm{TFR}^{2}$ \\
\hline \multirow[t]{2}{*}{ EAST MIDLANDS } & \multirow[t]{2}{*}{54,192} & \multirow[t]{2}{*}{61.0} & \multirow[t]{2}{*}{1.93} & Shropshire & 2,894 & 57.9 & 1.98 \\
\hline & & & & Bridgnorth & 428 & 50.4 & 1.75 \\
\hline Derby UA & 3,612 & 70.0 & 2.12 & North Shropshire & 652 & 62.7 & 2.19 \\
\hline Leicester UA & 5,176 & 73.3 & 2.12 & Oswestry & 400 & 53.2 & 1.80 \\
\hline Nottingham UA & 4,181 & 55.9 & 1.67 & Shrewsbury and Atcham & 1,071 & 61.9 & 2.07 \\
\hline Rutland UA & 355 & 53.9 & 2.35 & South Shropshire & 343 & 54.7 & 1.97 \\
\hline Derbyshire & 8,357 & 57.9 & 1.93 & Staffordshire & 9,084 & 57.9 & 1.92 \\
\hline Amber Valley & 1,259 & 55.0 & 1.82 & Cannock Chase & 1,144 & 58.3 & 1.92 \\
\hline Bolsover & 899 & 61.8 & 2.06 & East Staffordshire & 1,486 & 71.1 & 2.40 \\
\hline Chesterfield & 1,232 & 63.4 & 2.07 & Lichfield & 1,000 & 57.4 & 1.91 \\
\hline Derbyshire Dales & 554 & 49.6 & 1.78 & Newcastle-under-Lyme & 1,247 & 49.6 & 1.59 \\
\hline Erewash & 1,313 & 58.3 & 1.91 & South Staffordshire & 865 & 46.3 & 1.63 \\
\hline High Peak & 1,034 & 57.4 & 1.93 & Stafford & 1,386 & 61.1 & 2.02 \\
\hline North East Derbyshire & 924 & 53.0 & 1.79 & Staffordshire Moorlands & 916 & 54.8 & 1.92 \\
\hline South Derbyshire & 1,142 & 62.1 & 2.04 & Tamworth & 1,040 & 65.5 & 2.05 \\
\hline Leicestershire & 6,830 & 54.4 & 1.78 & Warwickshire & 6,241 & 61.6 & 1.95 \\
\hline Blaby & 1,038 & 58.8 & 1.90 & North Warwickshire & 647 & 54.6 & 1.82 \\
\hline Charnwood & 1,745 & 49.3 & 1.60 & Nuneaton and Bedworth & 1,664 & 69.5 & 2.22 \\
\hline Harborough & 818 & 55.1 & 1.88 & Rugby & 1,181 & 68.6 & 2.30 \\
\hline Hinckley and Bosworth & 1,092 & 54.9 & 1.76 & Stratford-on-Avon & 1,204 & 59.9 & 1.98 \\
\hline Melton & 531 & 58.6 & 1.99 & Warwick & 1,545 & 54.8 & 1.65 \\
\hline North West Leicestershire & 1,064 & 61.7 & 2.01 & West Midlands (Met County) & 39,559 & 71.3 & 2.16 \\
\hline \multirow[t]{2}{*}{ Oadby and Wigston } & \multirow[t]{2}{*}{542} & \multirow[t]{2}{*}{47.6} & \multirow[t]{2}{*}{1.76} & Birmingham & 17,311 & 75.2 & 2.22 \\
\hline & & & & Coventry & 4,618 & 69.2 & 2.06 \\
\hline Lincolnshire & 7,691 & 61.4 & 2.07 & Dudley & 3,707 & 62.5 & 2.01 \\
\hline Boston & 833 & 82.6 & 2.81 & Sandwell & 4,782 & 78.5 & 2.43 \\
\hline East Lindsey & 1,297 & 59.4 & 2.10 & Solihull & 2,171 & 55.8 & 1.84 \\
\hline Lincoln & 1,247 & 61.5 & 1.86 & & & & \\
\hline North Kesteven & 1,077 & 55.6 & 1.87 & Walsall & 3,634 & 72.0 & 2.29 \\
\hline South Holland & 918 & 66.0 & 2.32 & Wolverhampton & 3,336 & 69.2 & 2.11 \\
\hline South Kesteven & 1,448 & 59.9 & 2.05 & Worcestershire & 6,150 & 59.7 & 1.96 \\
\hline \multirow[t]{2}{*}{ West Lindsey } & \multirow[t]{2}{*}{871} & \multirow[t]{2}{*}{56.0} & \multirow[t]{2}{*}{1.98} & Bromsgrove & 904 & 54.5 & 1.83 \\
\hline & & & & Malvern Hills & 622 & 53.3 & 2.01 \\
\hline Northamptonshire & 9,225 & 66.6 & 2.13 & Redditch & 1,154 & 70.9 & 2.18 \\
\hline Corby & 880 & 77.9 & 2.57 & Worcester & 1,299 & 63.9 & 1.93 \\
\hline Daventry & 878 & 58.2 & 1.97 & Wychavon & 1,140 & 56.5 & 1.96 \\
\hline East Northamptonshire & 1,039 & 62.3 & 2.12 & Wyre Forest & 1,031 & 57.4 & 1.89 \\
\hline Kettering & 1,212 & 67.1 & 2.12 & & & & \\
\hline Northampton & 3,298 & 73.1 & 2.18 & EAST & 71,738 & 63.8 & 2.00 \\
\hline South Northamptonshire & 922 & 52.3 & 1.71 & Luton UA & 3,555 & 85.3 & 2.50 \\
\hline \multirow[t]{2}{*}{ Wellingborough } & \multirow[t]{2}{*}{996} & \multirow[t]{2}{*}{67.6} & \multirow[t]{2}{*}{2.20} & Peterborough UA & 2,970 & 85.8 & 2.63 \\
\hline & & & & Southend-on-Sea UA & 2,256 & 70.8 & 2.20 \\
\hline Nottinghamshire & 8,765 & 58.1 & 1.87 & Thurrock UA & 2,430 & 73.5 & 2.24 \\
\hline Ashfield & 1,439 & 60.8 & 1.97 & & & & \\
\hline Bassetlaw & 1,200 & 58.1 & 1.99 & Bedfordshire & 5,357 & 64.2 & 2.01 \\
\hline Broxtowe & 1,198 & 52.4 & 1.61 & Bedford & 2,111 & 65.5 & 2.00 \\
\hline Gedling & 1,195 & 54.8 & 1.77 & Mid Bedfordshire & 1,618 & 59.1 & 1.84 \\
\hline Mansfield & 1,396 & 69.5 & 2.24 & South Bedfordshire & 1,628 & 68.1 & 2.18 \\
\hline Newark and Sherwood & 1,199 & 57.4 & 1.92 & Cambridgeshire & 7,377 & 59.0 & 1.82 \\
\hline \multirow[t]{2}{*}{ Rushcliffe } & 1,138 & 54.2 & 1.69 & Cambridge & 1,421 & 43.6 & 1.44 \\
\hline & & & & East Cambridgeshire & 1,061 & 66.9 & 2.13 \\
\hline WEST MIDLANDS & 71,725 & 66.4 & 2.09 & Fenland & 1,056 & 63.9 & 2.14 \\
\hline & & & & Huntingdonshire & 2,036 & 60.6 & 1.96 \\
\hline Herefordshire, County of UA & 1,753 & 57.7 & 2.00 & South Cambridgeshire & 1,803 & 68.1 & 2.15 \\
\hline Stoke-on-Trent UA & 3.877 & 77.9 & 2.36 & & & & \\
\hline Telford and Wrekin UA & 2167 & 65.0 & 2.13 & & & & \\
\hline
\end{tabular}

1 The General Fertility Rate (GFR) is the number of live births per 1,000 women aged 15-44. The national GFRs have been calculated using 2006-based population projections for 2008 and the sub-national GFRs have been calculated using the 2007 mid-year population estimates.

2 The Total Fertility Rate (TFR) is the average number of live children that a group of women would bear if they experienced the age-specific fertility rates of the calendar year in question throughout their childbearing lifespan. The national TFRs have been calculated using the number of live births by single year of age and the 2006-based population projections for 2008 . The sub-national TFRs have been calculated using the number of live births by five year age groups and the 2007 mid-year population estimates.

3 City of London has been grouped with Hackney, and Isles of Scilly grouped with Penwith, because of the very small number of births in these areas. 
Table 2 continued

Live births by local authority of usual residence of mother, numbers, General Fertility Rates and Total Fertility Rates, 2008

England and Wales, Government Office Regions (within England), unitary authorities/counties/districts \& London boroughs

\begin{tabular}{|c|c|c|c|c|c|c|c|}
\hline Area of usual residence & Live births & $\mathrm{GFR}^{1}$ & $\mathrm{TFR}^{2}$ & - continued & Live births & $\mathrm{GFR}^{1}$ & $\mathrm{TFR}^{2}$ \\
\hline Essex & 16,005 & 59.6 & 1.89 & Southwark & 5,008 & 68.7 & 1.92 \\
\hline Basildon & 2,419 & 68.3 & 2.13 & Tower Hamlets & 4,230 & 69.3 & 1.82 \\
\hline Braintree & 1,732 & 63.0 & 2.05 & Wandsworth & 5,246 & 61.2 & 1.66 \\
\hline Brentwood & 761 & 55.6 & 1.78 & Westminster & 2,887 & 43.8 & 1.21 \\
\hline Castle Point & 832 & 52.7 & 1.78 & & & & \\
\hline \multirow{2}{*}{ Chelmsford } & 1,938 & 57.7 & 1.77 & Outer London & 74,907 & 73.1 & 2.14 \\
\hline & & & & Barking and Dagenham & 3,619 & 94.0 & 2.80 \\
\hline Colchester & 2,076 & 52.7 & 1.57 & Barnet & 5,195 & 71.0 & 2.03 \\
\hline Epping Forest & 1,500 & 61.9 & 1.96 & Bexley & 2,975 & 64.5 & 2.07 \\
\hline Harlow & 1,270 & 75.2 & 2.27 & Brent & 4,899 & 75.7 & 2.13 \\
\hline Maldon & 586 & 53.1 & 1.83 & Bromley & 3,983 & 64.6 & 1.98 \\
\hline \multirow[t]{2}{*}{ Rochford } & 776 & 51.4 & 1.72 & & & & \\
\hline & & & & Croydon & 5,331 & 70.3 & 2.16 \\
\hline Tendring & 1,307 & 57.1 & 1.93 & Ealing & 5,549 & 77.4 & 2.16 \\
\hline \multirow[t]{2}{*}{ Uttlesford } & 808 & 62.2 & 2.16 & Enfield & 5,000 & 79.4 & 2.38 \\
\hline & & & & Greenwich & 4,361 & 80.9 & 2.30 \\
\hline Hertfordshire & 14,496 & 65.5 & 2.01 & Harrow & 3,230 & 70.4 & 2.09 \\
\hline Broxbourne & 1,209 & 64.4 & 2.01 & & & & \\
\hline Dacorum & 1,881 & 68.9 & 2.18 & Havering & 2,787 & 62.0 & 1.98 \\
\hline East Hertfordshire & 1,634 & 58.9 & 1.78 & Hillingdon & 4,126 & 72.3 & 2.19 \\
\hline Hertsmere & 1,270 & 62.8 & 1.91 & Hounslow & 4,209 & 80.8 & 2.27 \\
\hline \multirow[t]{2}{*}{ North Hertfordshire } & 1,537 & 62.7 & 1.94 & Kingston upon Thames & 2,248 & 60.0 & 1.73 \\
\hline & & & & Merton & 3,330 & 68.6 & 1.88 \\
\hline St Albans & 2,064 & 77.7 & 2.29 & & & & \\
\hline Stevenage & 1,128 & 66.2 & 2.10 & Redbridge & 4,013 & 71.4 & 2.08 \\
\hline Three Rivers & 1,057 & 61.7 & 1.90 & Richmond upon Thames & 2,865 & 71.5 & 1.91 \\
\hline Watford & 1,354 & 75.5 & 2.18 & Sutton & 2,605 & 63.8 & 1.93 \\
\hline Welwyn Hatfield & 1,362 & 56.9 & 1.76 & Waltham Forest & 4,582 & 86.7 & 2.52 \\
\hline Norfolk & 9,057 & 59.0 & 1.87 & SOUTH EAST & 104,022 & 62.5 & 1.96 \\
\hline Breckland & 1,424 & 61.8 & 2.01 & & & & \\
\hline Broadland & 1,132 & 52.6 & 1.74 & Bracknell Forest UA & 1,573 & 61.9 & 1.88 \\
\hline Great Yarmouth & 1,136 & 67.7 & 2.26 & Brighton and Hove UA & 3,303 & 53.6 & 1.58 \\
\hline King's Lynn and West Norfolk & 1,576 & 64.4 & 2.14 & Isle of Wight UA & 1,270 & 55.1 & 1.90 \\
\hline \multirow[t]{2}{*}{ North Norfolk } & 834 & 57.1 & 1.97 & Medway UA & 3,419 & 64.2 & 2.03 \\
\hline & & & & Milton Keynes UA & 3,767 & 76.2 & 2.33 \\
\hline Norwich & 1,826 & 55.4 & 1.60 & & & & \\
\hline \multirow[t]{2}{*}{ South Norfolk } & 1,129 & 55.8 & 1.92 & Portsmouth UA & 2,653 & 55.5 & 1.63 \\
\hline & & & & Reading UA & 2,508 & 73.1 & 2.11 \\
\hline Suffolk & 8,235 & 62.7 & 2.03 & Slough UA & 2,592 & 94.0 & 2.68 \\
\hline Babergh & 838 & 55.8 & 1.92 & Southampton UA & 3,279 & 57.6 & 1.67 \\
\hline Forest Heath & 869 & 65.5 & 1.92 & West Berkshire UA & 2,022 & 67.3 & 2.22 \\
\hline Ipswich & 1,871 & 71.1 & 2.08 & & & & \\
\hline Mid Suffolk & 971 & 59.2 & 2.04 & Windsor and Maidenhead UA & 1,793 & 62.8 & 1.88 \\
\hline St Edmundsbury & 1,307 & 69.1 & 2.23 & Wokingham UA & 1,941 & 58.8 & 1.81 \\
\hline Suffolk Coastal & 1,110 & 53.1 & 1.85 & Buckinghamshire & 6,076 & 63.3 & 1.99 \\
\hline \multirow[t]{2}{*}{ Waveney } & 1,269 & 62.2 & 2.09 & Aylesbury Vale & 2,122 & 59.5 & 1.87 \\
\hline & & & & Chiltern & $\begin{array}{r}2,1<2 \\
935\end{array}$ & 57.4 & 1.88 \\
\hline \multirow[t]{2}{*}{ LONDON } & 127,651 & 69.4 & 1.95 & South Bucks & 694 & 58.9 & 1.90 \\
\hline & & & & Wycombe & 2,325 & 71.9 & 2.21 \\
\hline Inner London & 52,744 & 64.7 & 1.78 & & & & \\
\hline Camden & 3,061 & 45.2 & 1.26 & East Sussex & 5,157 & 60.1 & 2.04 \\
\hline Hackney plus City of London ${ }^{3}$ & 4,485 & 77.5 & 2.19 & Eastbourne & 1,092 & 61.6 & 1.93 \\
\hline Hammersmith and Fulham & 2,733 & 57.4 & 1.58 & Hastings & 1,153 & 68.5 & 2.21 \\
\hline \multirow[t]{2}{*}{ Haringey } & 4,289 & 74.8 & 2.12 & Lewes & 910 & 58.4 & 1.99 \\
\hline & & & & Rother & 698 & 55.7 & 2.07 \\
\hline Islington & 2,917 & 53.6 & 1.51 & Wealden & 1,304 & 56.2 & 2.02 \\
\hline Kensington and Chelsea & 2,216 & 49.9 & 1.33 & & & & \\
\hline Lambeth & 4,837 & 66.0 & 1.86 & Hampshire & 14,676 & 60.3 & 1.96 \\
\hline Lewisham & 4,872 & 74.3 & 2.14 & Basingstoke and Deane & 2,059 & 62.0 & 1.92 \\
\hline \multirow[t]{2}{*}{ Newham } & 5,963 & 96.5 & 2.68 & East Hampshire & 1,177 & 60.0 & 2.12 \\
\hline & & & & Eastleigh & 1,432 & 58.9 & 1.85 \\
\hline
\end{tabular}

1 The General Fertility Rate (GFR) is the number of live births per 1,000 women aged 15-44. The national GFRs have been calculated using 2006 -based population projections for 2008 and the sub-national GFRs have been calculated using the 2007 mid-year population estimates.

2 The Total Fertility Rate (TFR) is the average number of live children that a group of women would bear if they experienced the age-specific fertility rates of the calendar year in question throughout their childbearing lifespan. The national TFRs have been calculated using the number of live births by single year of age and the 2006 -based population projections for 2008 . The sub-national TFRs have been calculated using the number of live births by five year age groups and the 2007 mid-year population estimates.

3 City of London has been grouped with Hackney, and Isles of Scilly grouped with Penwith, because of the very small number of births in these areas. 
England and Wales, Government Office Regions (within England), unitary authorities/counties/districts \& London boroughs

\begin{tabular}{|c|c|c|c|c|c|c|c|}
\hline Area of usual residence & Live births & $\mathrm{GFR}^{1}$ & $\mathrm{TFR}^{2}$ & - continued & Live births & $\mathrm{GFR}^{1}$ & $\mathrm{TFR}^{2}$ \\
\hline Fareham & 1,128 & 57.9 & 2.03 & North Somerset UA & 2,268 & 62.1 & 2.03 \\
\hline Gosport & 1,101 & 69.1 & 2.14 & Plymouth UA & 3,216 & 59.9 & 1.86 \\
\hline Hart & 1,096 & 61.8 & 1.91 & Poole UA & 1,629 & 63.7 & 2.07 \\
\hline Havant & 1,265 & 59.7 & 2.00 & South Gloucestershire UA & 3,133 & 60.1 & 1.92 \\
\hline New Forest & 1,595 & 55.4 & 1.92 & Swindon UA & 2,843 & 71.1 & 2.20 \\
\hline Rushmoor & 1,327 & 66.4 & 2.00 & Torbay UA & 1,422 & 62.4 & 2.07 \\
\hline Test Valley & 1,308 & 59.5 & 1.97 & & & & \\
\hline \multirow[t]{2}{*}{ Winchester } & 1,188 & 56.4 & 1.86 & Cornwall and Isles of Scilly & 5,442 & 58.4 & 1.93 \\
\hline & & & & Caradon & 810 & 57.9 & 2.01 \\
\hline Kent & 17,095 & 63.1 & 2.03 & Carrick & 833 & 49.2 & 1.59 \\
\hline Ashford & 1,528 & 69.9 & 2.33 & Kerrier & 1,106 & 61.8 & 2.04 \\
\hline Canterbury & 1,481 & 46.1 & 1.53 & North Cornwall & 890 & 61.2 & 2.09 \\
\hline Dartford & 1,313 & 66.2 & 2.01 & Penwith plus Isles of Scilly ${ }^{3}$ & 607 & 54.5 & 1.82 \\
\hline Dover & 1,204 & 62.8 & 2.15 & Restormel & 1,196 & 63.9 & 2.06 \\
\hline \multirow[t]{2}{*}{ Gravesham } & 1,226 & 61.9 & 1.97 & & & & \\
\hline & & & & Devon & 7,172 & 54.3 & 1.80 \\
\hline Maidstone & 1,753 & 63.1 & 1.99 & East Devon & 1,063 & 53.2 & 1.92 \\
\hline Sevenoaks & 1,331 & 62.1 & 1.99 & Exeter & 1,411 & 47.3 & 1.45 \\
\hline Shepway & 1,195 & 66.9 & 2.22 & Mid Devon & 810 & 60.7 & 2.08 \\
\hline Swale & 1,712 & 67.4 & 2.21 & North Devon & 965 & 61.9 & 2.15 \\
\hline Thanet & 1,600 & 69.1 & 2.25 & South Hams & 677 & 50.6 & 1.81 \\
\hline Tonbridge and Malling & 1,342 & 59.3 & 1.93 & Teignbridge & 1,143 & 53.9 & 1.87 \\
\hline \multirow[t]{2}{*}{ Tunbridge Wells } & 1,410 & 70.4 & 2.40 & Torridge & 615 & 57.8 & 1.99 \\
\hline & & & & West Devon & 488 & 59.9 & 2.20 \\
\hline Oxfordshire & 8,307 & 61.3 & 1.89 & & & & \\
\hline Cherwell & 1,955 & 68.7 & 2.16 & Dorset & 3,734 & 58.8 & 2.09 \\
\hline Oxford & 2,006 & 47.8 & 1.55 & Christchurch & 390 & 58.7 & 2.07 \\
\hline South Oxfordshire & 1,610 & 66.4 & 2.11 & East Dorset & 689 & 55.4 & 2.13 \\
\hline Vale of White Horse & 1,451 & 66.1 & 2.10 & North Dorset & 690 & 61.6 & 2.16 \\
\hline \multirow[t]{2}{*}{ West Oxfordshire } & 1,285 & 67.9 & 2.25 & Purbeck & 413 & 55.0 & 1.88 \\
\hline & & & & West Dorset & 838 & 57.6 & 2.18 \\
\hline Surrey & 13,710 & 62.3 & 1.90 & Weymouth and Portland & 714 & 64.1 & 2.13 \\
\hline Elmbridge & 1,783 & 68.0 & 1.98 & & & & \\
\hline Epsom and Ewell & 904 & 62.8 & 1.92 & Gloucestershire & 6,730 & 60.8 & 2.00 \\
\hline Guildford & 1,629 & 56.3 & 1.72 & Cheltenham & 1,361 & 57.3 & 1.80 \\
\hline Mole Valley & 837 & 59.9 & 1.97 & Cotswold & 760 & 53.1 & 1.79 \\
\hline \multirow[t]{2}{*}{ Reigate and Banstead } & 1,768 & 66.7 & 2.01 & Forest of Dean & 817 & 57.0 & 1.98 \\
\hline & & & & Gloucester & 1,746 & 71.8 & 2.29 \\
\hline Runnymede & 969 & 50.5 & 1.56 & Stroud & 1,137 & 58.4 & 2.00 \\
\hline Spelthorne & 1,163 & 63.4 & 1.99 & Tewkesbury & 909 & 62.8 & 2.04 \\
\hline Surrey Heath & 1,029 & 62.9 & 2.00 & & & & \\
\hline Tandridge & 927 & 60.5 & 2.02 & Somerset & 5,614 & 60.9 & 2.08 \\
\hline Waverley & 1,303 & 60.2 & 1.92 & Mendip & 1,176 & 58.8 & 2.04 \\
\hline \multirow[t]{2}{*}{ Woking } & 1,398 & 72.3 & 2.11 & Sedgemoor & 1,152 & 58.4 & 2.02 \\
\hline & & & & South Somerset & 1,771 & 64.8 & 2.20 \\
\hline West Sussex & 8,881 & 63.1 & 2.05 & Taunton Deane & 1,243 & 61.9 & 2.03 \\
\hline Adur & 646 & 59.7 & 1.95 & West Somerset & 272 & 54.2 & 2.08 \\
\hline Arun & 1,518 & 64.8 & 2.20 & & & & \\
\hline Chichester & 1,091 & 60.1 & 2.03 & Wiltshire & 5,421 & 65.0 & 2.22 \\
\hline Crawley & 1,509 & 69.4 & 2.07 & Kennet & 935 & 66.2 & 2.43 \\
\hline \multirow[t]{2}{*}{ Horsham } & 1,313 & 54.9 & 1.81 & North Wiltshire & 1,592 & 62.9 & 2.16 \\
\hline & & & & Salisbury & 1,371 & 65.9 & 2.20 \\
\hline Mid Sussex & 1,587 & 66.1 & 2.12 & West Wiltshire & 1,523 & 65.8 & 2.21 \\
\hline \multirow[t]{2}{*}{ Worthing } & 1,217 & 65.0 & 2.05 & & & & \\
\hline & & & & WALES & 35,649 & 61.7 & 1.96 \\
\hline \multirow[t]{2}{*}{ SOUTH WEST } & 58,742 & 60.0 & 1.92 & & & & \\
\hline & & & & Isle of Anglesey & 780 & 65.0 & 2.13 \\
\hline Bath and North East & & & & Gwynedd & 1,275 & 57.7 & 1.83 \\
\hline Somerset UA & 1,768 & 47.7 & 1.56 & Conwy & 1,163 & 63.3 & 2.15 \\
\hline Bournemouth UA & 2,095 & 60.4 & 1.81 & Denbighshire & 1,076 & 63.1 & 2.10 \\
\hline Bristol, City of UA & 6,255 & 61.2 & 1.80 & Flintshire & 1,743 & 59.9 & 1.97 \\
\hline
\end{tabular}

1 The General Fertility Rate (GFR) is the number of live births per 1,000 women aged 15-44. The national GFRs have been calculated using 2006-based population projections for 2008 and the sub-national GFRs have been calculated using the 2007 mid-year population estimates.

2 The Total Fertility Rate (TFR) is the average number of live children that a group of women would bear if they experienced the age-specific fertility rates of the calendar year in question throughout their childbearing lifespan. The national TFRs have been calculated using the number of live births by single year of age and the 2006 -based population projections for 2008 . The sub-national TFRs have been calculated using the number of live births by five year age groups and the 2007 mid-year population estimates.

3 City of London has been grouped with Hackney, and Isles of Scilly grouped with Penwith, because of the very small number of births in these areas. 
England and Wales, Government Office Regions (within England), unitary authorities/counties/districts \& London boroughs

\begin{tabular}{|c|c|c|c|c|c|c|c|}
\hline Area of usual residence & Live births & $\mathrm{GFR}^{1}$ & $\mathrm{TFR}^{2}$ & - continued & Live births & $\mathrm{GFR}^{1}$ & $\mathrm{TFR}^{2}$ \\
\hline Wrexham & 1,793 & 69.1 & 2.18 & Rhondda, Cynon, Taff & 2,908 & 61.0 & 1.89 \\
\hline Powys & 1,280 & 60.3 & 2.14 & Merthyr Tydfil & 781 & 70.6 & 2.30 \\
\hline Ceredigion & 603 & 40.0 & 1.43 & Caerphilly & 2,223 & 64.7 & 2.06 \\
\hline Pembrokeshire & 1,251 & 61.8 & 2.10 & Blaenau Gwent & 867 & 63.0 & 2.04 \\
\hline Carmarthenshire & 1,971 & 61.3 & 2.01 & Torfaen & 1,056 & 60.5 & 1.96 \\
\hline Swansea & 2,732 & 60.3 & 1.90 & Monmouthshire & 919 & 60.9 & 2.20 \\
\hline Neath Port Talbot & 1,551 & 60.1 & 1.96 & Newport & 1,999 & 70.3 & 2.30 \\
\hline Bridgend & 1,630 & 62.9 & 2.07 & & & & \\
\hline The Vale of Glamorgan & 1,482 & 62.4 & 2.04 & Normal residence outside & & & \\
\hline Cardiff & 4,566 & 59.1 & 1.82 & England and Wales & 252 & - & - \\
\hline
\end{tabular}

1 The General Fertility Rate (GFR) is the number of live births per 1,000 women aged 15-44. The national GFRs have been calculated using 2006-based population projections for 2008 and the sub-national GFRs have been calculated using the 2007 mid-year population estimates.

2 The Total Fertility Rate (TFR) is the average number of live children that a group of women would bear if they experienced the age-specific fertility rates of the calendar year in question throughout their childbearing lifespan. The national TFRs have been calculated using the number of live births by single year of age and the 2006 -based population projections for 2008 . The sub-national TFRs have been calculated using the number of live births by five year age groups and the 2007 mid-year population estimates.

3 City of London has been grouped with Hackney, and Isles of Scilly grouped with Penwith, because of the very small number of births in these areas.

\section{Table 3} Live births by health area of usual residence of mother, numbers, General Fertility Rates and Total Fertility Rates, 2008

England and Wales, Government Office Regions (within England), and health authorities/boards ${ }^{1}$

\begin{tabular}{|c|c|c|c|c|c|c|c|}
\hline Area of usual residence & Live births & $\mathrm{GFR}^{2}$ & $\mathrm{TFR}^{3}$ & - continued & Live births & $\mathrm{GFR}^{2}$ & $\mathrm{TFR}^{3}$ \\
\hline \multirow[t]{2}{*}{ ENGLAND AND WALES } & 708,708 & 63.5 & 1.95 & WALES & 35,649 & 61.7 & 1.96 \\
\hline & & & & Anglesey & 780 & 65.0 & 2.13 \\
\hline \multirow[t]{2}{*}{ ENGLAND } & 672,807 & 63.6 & 1.95 & Gwynedd & 1,275 & 57.7 & 1.83 \\
\hline & & & & Conwy & 1,163 & 63.3 & 2.15 \\
\hline NORTH EAST & 30,217 & 58.7 & 1.86 & Denbighshire & 1,076 & 63.1 & 2.10 \\
\hline North East & 30,217 & 58.7 & 1.86 & Flintshire & 1,743 & 59.9 & 1.97 \\
\hline NORTH WEST & 88,167 & 63.2 & 2.00 & Wrexham & 1,793 & 69.1 & 2.18 \\
\hline \multirow[t]{2}{*}{ North West } & 88,167 & 63.2 & 2.00 & Powys Teaching & 1,280 & 60.3 & 2.14 \\
\hline & & & & Ceredigion & 603 & 40.0 & 1.43 \\
\hline YORKSHIRE AND THE HUMBER & 66,353 & 62.4 & 1.94 & Pembrokeshire & 1,251 & 61.8 & 2.10 \\
\hline Yorkshire and The Humber & 66,353 & 62.4 & 1.94 & Carmarthenshire & 1,971 & 61.3 & 2.01 \\
\hline EAST MIDLANDS & 54,192 & 61.0 & 1.93 & Swansea & 2,732 & 60.3 & 1.90 \\
\hline \multirow[t]{2}{*}{ East Midlands } & 54,192 & 61.0 & 1.93 & Neath Port Talbot & 1,551 & 60.1 & 1.96 \\
\hline & & & & Bridgend & 1,630 & 62.9 & 2.07 \\
\hline WEST MIDLANDS & 71,725 & 66.4 & 2.09 & Vale of Glamorgan & 1,482 & 62.4 & 2.04 \\
\hline West Midlands & 71,725 & 66.4 & 2.09 & Cardiff & 4,566 & 59.1 & 1.82 \\
\hline EAST & 71,738 & 63.8 & 2.00 & Rhondda Cynon Taff Teaching & 2,908 & 61.0 & 1.89 \\
\hline \multirow[t]{2}{*}{ East of England } & 71,738 & 63.8 & 2.00 & Merthyr Tydfil & 781 & 70.6 & 2.30 \\
\hline & & & & Caerphilly Teaching & 2,223 & 64.7 & 2.06 \\
\hline LONDON & 127,651 & 69.4 & 1.95 & Blaenau Gwent & 867 & 63.0 & 2.04 \\
\hline London & 127,651 & 69.4 & 1.95 & Torfaen & 1,056 & 60.5 & 1.96 \\
\hline SOUTH EAST & 104,022 & 62.5 & 1.96 & Monmouthshire & 919 & 60.9 & 2.20 \\
\hline South East Coast & 51,565 & 61.9 & 1.96 & Newport & 1,999 & 70.3 & 2.30 \\
\hline \multirow{2}{*}{ South Central } & 52,457 & 63.1 & 1.95 & & & & \\
\hline & & & & Normal residence outside & 252 & - & - \\
\hline SOUTH WEST & 58,742 & 60.0 & 1.92 & England and Wales & & & \\
\hline South West & 58,742 & 60.0 & 1.92 & & & & \\
\hline
\end{tabular}

Notes: Figures for 2008 are provisional. A birth to a mother whose usual residence is outside England and Wales is assigned to the country of residence. These births are included in total figures for England and Wales, but excluded from any sub-division of England and Wales.

1 Strategic Health Authorities in England and Local Health Boards in Wales.

2 The General Fertility Rate (GFR) is the number of live births per 1,000 women aged 15-44. The national GFRs have been calculated using 2006-based population projections for 2008 and the sub-national GFRs have been calculated using the 2007 mid-year population estimates.

3 The Total Fertility Rate (TFR) is the average number of live children that a group of women would bear if they experienced the age-specific fertility rates of the calendar year in question throughout their childbearing lifespan. The national TFRs have been calculated using the number of live births by single year of age and the 2006-based population projections for 2008 . The sub-national TFRs have been calculated using the number of live births by five year age groups and the 2007 mid-year population estimates. 